\title{
Molecular and Cellular Biomarkers of COVID-19 Prognosis: Protocol for the Prospective Cohort TARGET Study
}

Patricia Kurizky ${ }^{1,2^{*}}, \mathrm{MD}, \mathrm{PhD}$; Otávio T Nóbrega ${ }^{1^{*}}, \mathrm{PhD}$; Alexandre Anderson De Sousa Munhoz Soares ${ }^{1^{*}}, \mathrm{MD}$ $\mathrm{PhD}$; Rodrigo Barbosa Aires ${ }^{1 *}, \mathrm{MD}$; Cleandro Pires De Albuquerque ${ }^{1,2^{*}}, \mathrm{MSc}, \mathrm{MD}$; André Moraes Nicola ${ }^{1^{*}}, \mathrm{MD}$, $\mathrm{PhD}$; Patrícia Albuquerque ${ }^{3 *}, \mathrm{PhD}$; Andréa Teixeira-Carvalho ${ }^{4 *}, \mathrm{PhD}$; Luciana Ansaneli Naves ${ }^{1,2^{*}}, \mathrm{MD}, \mathrm{PhD}$; Wagner Fontes $^{5 *}$, MD, PhD; Isabelle Souza Luz ${ }^{5}$, BS; Liza Felicori ${ }^{6 *}$, PhD; Ana Paulo Monteiro Gomides ${ }^{7 *}, \mathrm{MD}, \mathrm{PhD}$; Dayde Lane Mendonça-Silva ${ }^{2,8^{*}}, \mathrm{PhD}$; Laila Salmen Espindola ${ }^{1,8^{*}}, \mathrm{PhD}$; Olindo Assis Martins-Filho ${ }^{4 *}$, PhD; Sheila Maria Barbosa de Lima $^{9 *}, \mathrm{PhD}$; Licia Maria Henrique Mota ${ }^{1,2^{*}}, \mathrm{MD}, \mathrm{PhD}$; Ciro Martins Gomes ${ }^{1,2^{*}}, \mathrm{MD}, \mathrm{PhD}$

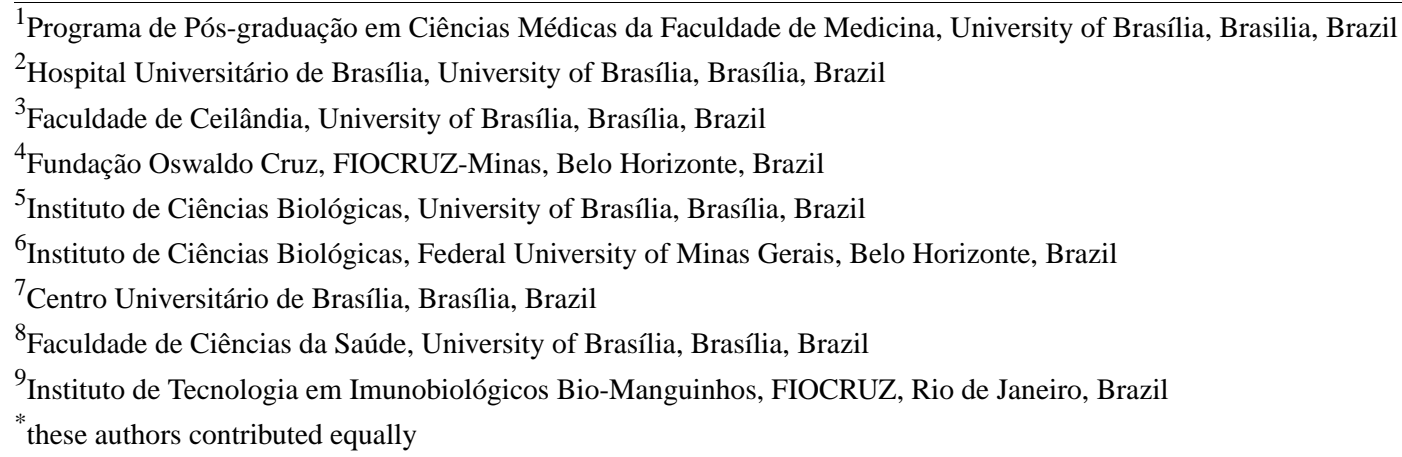

\section{Corresponding Author:}

Patricia Kurizky, MD, PhD

Programa de Pós-graduação em Ciências Médicas da Faculdade de Medicina

University of Brasília

Laboratório de Dermatomicologia - Faculdade de Medicina da UnB

Campus Universitário Darcy Ribeiro

Brasilia, 70910-900

Brazil

Phone: 5561981148432

Email: patyshu79@gmail.com

\section{Abstract}

Background: Since the beginning of the COVID-19 pandemic, the world's attention has been focused on better understanding the relation between the human host and the SARS-CoV-2 virus, as its action has led to hundreds of thousands of deaths.

Objective: In this context, we decided to study certain consequences of the abundant cytokine release over the innate and adaptive immune systems, inflammation, and hemostasis, comparing mild and severe forms of COVID-19.

Methods: To accomplish these aims, we will analyze demographic characteristics, biochemical tests, immune biomarkers, leukocyte phenotyping, immunoglobulin profile, hormonal release (cortisol and prolactin), gene expression, thromboelastometry, neutralizing antibodies, metabolic profile, and neutrophil function (reactive oxygen species production, neutrophil extracellular trap production, phagocytosis, migration, gene expression, and proteomics). A total of 200 reverse transcription polymerase chain reaction-confirmed patients will be enrolled and divided into two groups: mild/moderate or severe/critical forms of COVID-19. Blood samples will be collected at different times: at inclusion and after 9 and 18 days, with an additional 3-day sample for severe patients. We believe that this information will provide more knowledge for future studies that will provide more robust and useful clinical information that may allow for better decisions at the front lines of health care.

Results: The recruitment began in June 2020 and is still in progress. It is expected to continue until February 2021. Data analysis is scheduled to start after all data have been collected. The coagulation study branch is complete and is already in the analysis phase. 
Conclusions: This study is original in terms of the different parameters analyzed in the same sample of patients with COVID-19. The project, which is currently in the data collection phase, was approved by the Brazilian Committee of Ethics in Human Research (CAAE 30846920.7.0000.0008).

Trial Registration: Brazilian Registry of Clinical Trials RBR-62zdkk; https://ensaiosclinicos.gov.br/rg/RBR-62zdkk International Registered Report Identifier (IRRID)： DERR1-10.2196/24211

(JMIR Res Protoc 2021;10(3):e24211) doi: 10.2196/24211

\section{KEYWORDS}

COVID-19; TARGET; cytokine profile; neutrophil function; thromboelastometry; neutralizing antibodies; metabolomics; proteomics; biomarker; prognosis; design; cohort; virus; immunology; immune system; genetics

\section{Introduction}

COVID-19 emerged in the city of Wuhan, Hubei, China and spread worldwide over the next several months [1]. Originally reported by the World Health Organization (WHO) as a pneumonia outbreak of undetermined origin, COVID-19 had its epidemiological status revised by the WHO as a Public Health Emergency of International Concern by the end of January 2020 and as a pandemic at the beginning of March 2020 [2]. The etiologic agent was identified as a new coronavirus of the Betacoronavirus genus, named SARS-CoV-2, due to its structural similarity to severe acute respiratory syndrome-related coronavirus (SARS-CoV), which also accounted for an outbreak in China in 2002-2003 [3]. The disease is characterized by fever, cough, and dyspnea, and can progress to pulmonary failure and lead to liver, heart, and kidney damage. Clinical management of severe cases requires respiratory assistance [4].

Angiotensin converting enzyme-2 (ACE2) mediates SARS-CoV-2 (and other coronaviruses) entry via clathrin-mediated endocytosis into type-2 pneumocytes and macrophages of the lung milieu [5,6]. ACE2 is a membrane-bound enzyme that converts active angiotensin II to inactive angiotensin (1-7). Thus, ACE2 blocks the unfavorable effects of angiotensin II action, including classic vasoconstriction in addition to inflammation and thrombosis. SARS-CoV-2 binding markedly impairs ACE2 catalytic activity by competitive inhibition, with increased in situ pulmonary inflammation and coagulation as detrimental outcomes of weakened counter regulation of the angiotensin II/AT1 receptor axis [7].

Hence, COVID-19 resembles a hyperimmune syndrome defined by potentially lethal hypercytokinemia due to overproduction of a set of proinflammatory mediators, including interleukins (IL-1 $\beta$, IL-2, IL7), granulocyte colony stimulating factor, interferon-gamma (IFN- $\gamma$ ), monocyte chemoattractant protein-1 (MCP-1), and tumor necrosis factor-alpha (TNF- $\alpha$ ), which culminates in multi-organ failure [8,9]. In addition, the Th17-type response plays an important role in severe cases due to an additional proinflammatory overflow resulting from supraphysiological IL-1 $\beta$, IL-6, and TNF- $\alpha$ levels that contribute to edema formation [10]. The role of cortisol and prolactin in the modulation of the immune system has long been described $[11,12]$, but their participation (if any) in the COVID-19-related "cytokine storm" remains unknown. Moreover, given the intimate interplay of inflammation with blood clotting, severe patients usually show a hypercoagulable profile that leads to an increased frequency of pulmonary thromboembolism, deep vein thrombosis, and even heart and brain ischemia events $[13,14]$.

In this context, it is also no surprise that the first treatment that reduced COVID-19 mortality, in a large randomized controlled trial (RECOVERY [Randomised Evaluation of COVID-19 Therapy]) [12,15], was dexamethasone, a corticosteroid [12]. However, it also showed that the effect seems to depend on the clinical stage of the disease, highlighting the need for further investigations into the course of the inflammatory response. Other anti-inflammatory treatments such as tocilizumab, an IL-6 receptor-blocking humanized antibody used to treat rheumatoid arthritis, were used (out of compassion) in patients with severe COVID-19 in China and Italy [16-18], with some clinical improvement. Similarly, another drug under evaluation is baricitinib, a JAK2 inhibitor approved for myeloproliferative neoplasms and rheumatoid arthritis that reduces Th17-type cytokine secretion by blocking AP2-associated protein kinase 1 [19]. However, the clinical efficacy of baricitinib against COVID-19 has yet to be proven. These studies support the assumption that a cytokine "storm" not only plays a role in COVID-19 pathophysiology but also has predictive prognostic value to monitor the evolution of mild and severe cases.

New metabolomics protocols can characterize metabolic pathways and provide a broad view of the impact of SARS-CoV-2 on the body. Both metabolome and proteome composition are dynamic and reflect genome expression under specific conditions [20-22]. Furthermore, these innovative combination protocols can help in understanding the metabolic profile of a given patient or group of patients [23] from urine, saliva, and blood samples [24]. The chemical profile substantially contributed to biological and medical research, leading to advancements in clinical medical practice [23].

This paper describes the design and rationale of a multicenter prospective cohort study aimed at evaluating the molecular and cellular immune signature of COVID-19 by assessing serum inflammatory mediators and regulatory chemokines of Brazilian patients with different clinical forms of the disease.

\section{Methods}

\section{Study Design and Population}

TARGET is registered in the Brazilian Clinical Trials database with registry code RBR-62zdkk and constitutes a multicenter, prospective cohort study of consecutive COVID-19 cases, 
consisting of participants recruited from secondary and tertiary health care facilities in Brazil, namely, the University Hospital of Brasília (University of Brasília [UnB]) and the public regional hospitals of Asa Norte, Gama, Santa Maria, and Taguatinga (Brasília, Distrito Federal). Clinical procedures will be conducted according to a standard protocol approved by local institutional review boards under accession number CAAE 30846920.7.0000.0008, with participants included after voluntary signing of an informed consent form. The study protocol follows the recommendations of the STROBE (Strengthening the Reporting of Observational Studies in Epidemiology) statement for observational studies [16].

\section{Recruitment}

Inclusion criteria will include the following: laboratory confirmation of COVID-19 by reverse transcription polymerase chain reaction (RT-PCR; KIT Molecular SARS-CoV2 [E/RP]; Bio-Manguinhos), a signed informed consent form, and older than 18 years. The exclusion criteria are as follows: patients with inconclusive, unavailable, or solely serological laboratory diagnosis of COVID-19; a dementia condition (Alzheimer, Parkinson, vascular frontotemporal, Lewy body, or others); schizophrenia or schizoaffective disorders with psychotic characteristics; and patients with previously known congenital hemorrhagic diseases or thrombophilia or use of anticoagulants (for coagulation tests; Figure 1).

Figure 1. Inclusion and exclusion criteria flowchart. The T-branch group is patients referred to the emergency room with mild or moderate forms of COVID-19 not requiring hospitalization. The D-branch group is patients referred to the emergency room with severe or critical forms of COVID-19 requiring hospitalization.

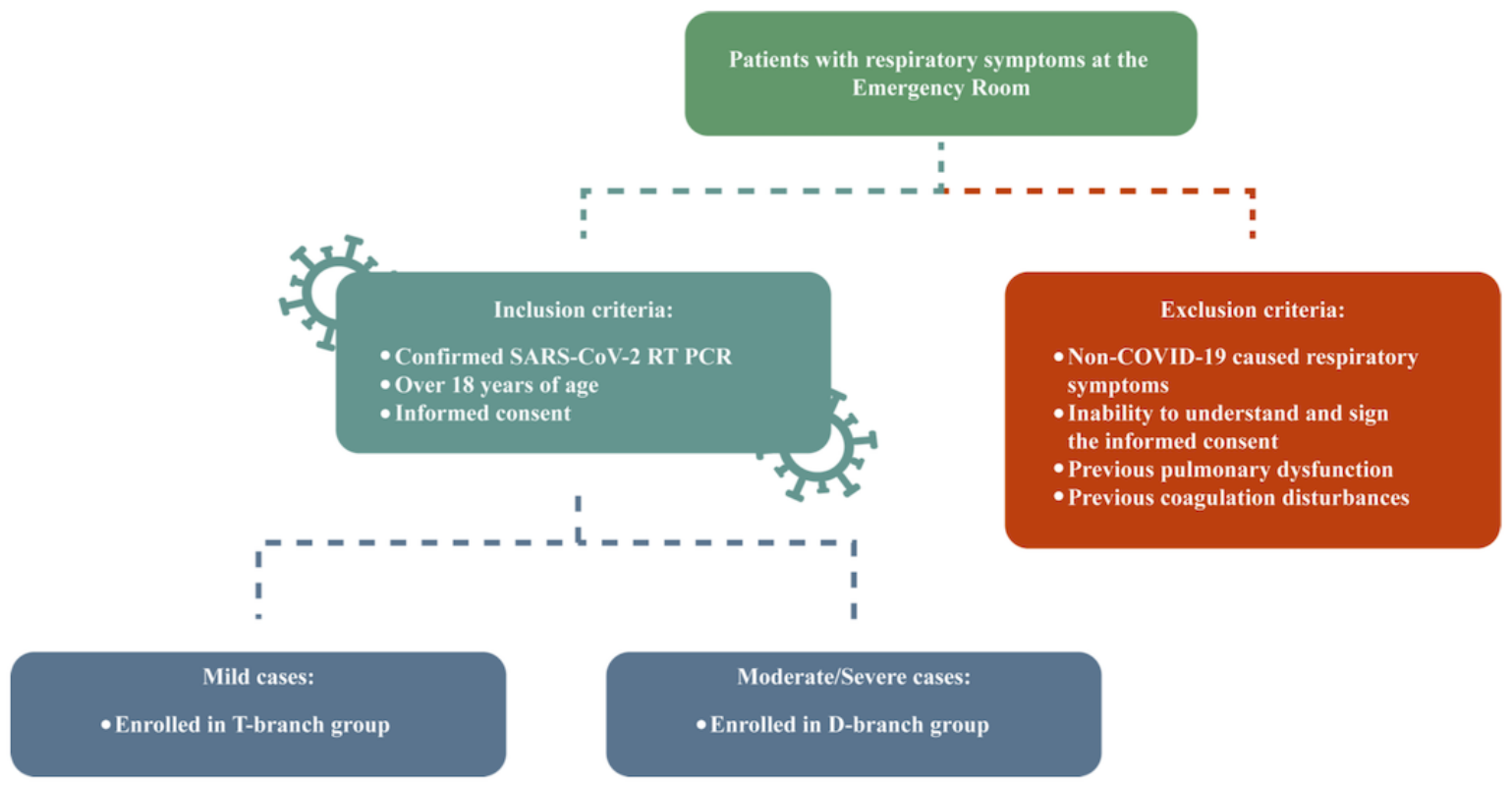

\section{Clinical Assessment Protocol}

The clinical evaluation aims to identify the signs and symptoms associated with COVID-19 and monitor the status (mild/moderate or severe/critical) of the disease. Clinical assessments include identification of comorbidities, COVID-19-associated symptoms and treatment, use of medications in general, clinical chemistry findings, and life support regimen care. Severe cases are defined for the purposes of the study by dyspnea (respiratory rate $>30$ breaths per minute) coupled with any of the following criteria: pulsed wave oxygen saturation $<93 \%$ at rest, $\mathrm{PaO}_{2} / \mathrm{FiO}_{2} \leq 300 \mathrm{mmHg}$, respiratory failure requiring mechanical ventilation, multiple organ failure, shock, or admission to the intensive care unit. Patients who do not meet the criteria for severe/critical forms will be considered mild/moderate cases. No asymptomatic patients will be included (Textbox 1 and Table 1). 
Textbox 1. Components being assessed for characterization of the following domains: symptoms, comorbidities, treatments, biochemical profile, immune biomarkers, and leukocyte phenotype and functionality in the TARGET protocol.

Symptoms
Fever, chills, sneezing, sore throat, headache, cough, coryza, anosmia, dysgeusia, diarrhea, asthenia, nausea, vomit, dizziness, and others
Comorbidities

Pulmonary chronic disease, chronic cardiopathy, hypertension, diabetes, chronic kidney disease, pregnancy, neoplasms, smoking, alcohol ingestion, psoriasis, immunodepression, HIV, Dengue, hanseniasis, Zika virus, chikungunya, Chagas disease, yellow fever, leishmaniasis, malaria, H1N1, and others

\section{Previous medications}

Angiotensin converting enzyme inhibitors, angiotensin II receptor antagonists, nonsteroidal anti-inflammatory drugs, and others

Treatments aimed at COVID-19

Clinical support, dexamethasone, enoxaparin, chloroquine, antibiotics, ivermectin, nitazoxanide, lopinavir, ritonavir, remdesivir, immunoglobulin, plasmapheresis, anti-IL6, JAK inhibitor, and others

\section{Biochemical tests}

Leukocyte, lymphocyte and platelet counts, ferritin, lactate dehydrogenase, troponin, creatine kinase, aspartate aminotransferase, alanine aminotransferase, and creatinine

Hormone evaluation

Prolactin and cortisol

Thorax computed tomography

Ground-glass opacity $(<25 \%, 25-50 \%,>50 \%)$

Consolidation

Serum immune biomarkers

Chemokines: CXCL8, CCL11, CCL3, CCL4, CCL2, CCL5, and CXCL10; inflammatory cytokines: IL-1 $\beta$, IL-6, TNF- $\alpha$, IL-12p70, IFN-gamma, IL-17A, and IL-15; regulatory cytokines: IL-1Ra, IL-4, IL-5, IL-9, IL-10, and IL-13; and cell growth factors: IL-2, IL-7, FGF-basic, PDGF, VEGF, G-CSF, and GM-CSF

\section{Gene expression assays}

IFN- $\gamma$, IL-12, IL-17a, TNF- $\alpha$, IL-6, IL-4, IL-5, IL-10, IL-1b, RANTES, MCP-1, MIP, MIG, IP-10, and IL-8

Leukocyte phenotyping

CD3, CD4, CD8, HLA-DR, CD25, CD19, CD5, CD27, CD38, PD-1, CD28, CD14, CD16, and CD56

Leukocyte functionality

IL-1 $\beta$, IL-6, TNF- $\alpha$, IFN- $\alpha$, IL-5-PE, and IL-10

Metabolomic analysis

Metabolic profile/pathways

Neutrophils

Isolation, reactive oxygen species production, phagocytosis, neuroendocrine tumors production, migration assay, neutrophil proteomics 
Table 1. The TARGET protocol will collect information about a series of characteristics and tests along the clinical evolution of patients with COVID-19. At each time point, the following data will be assessed.

\begin{tabular}{|c|c|c|c|c|}
\hline Data registered & Day 0 & Day 3 & Day 9 & Day 18 \\
\hline Age, gender, weight, and height & $\mathfrak{J}^{\mathrm{a}}$ & $-{ }^{b}$ & - & - \\
\hline Symptoms & $\checkmark$ & $\checkmark$ & $\checkmark$ & $\checkmark$ \\
\hline Contact with SARS-CoV-2 patient & $\checkmark$ & - & - & - \\
\hline Reverse transcription polymerase chain reaction & $\checkmark$ & - & - & - \\
\hline Time of symptoms & $\checkmark$ & - & - & - \\
\hline Comorbidities & $\checkmark$ & - & - & - \\
\hline Medications in use & $\checkmark$ & $\checkmark$ & $\checkmark$ & $\checkmark$ \\
\hline $\mathrm{SpO} 2$ & $\checkmark$ & $\checkmark$ & $\checkmark$ & $\checkmark$ \\
\hline Thorax computed tomography & $\checkmark$ & $\checkmark$ & $\checkmark$ & $\checkmark$ \\
\hline Biochemical tests & $\checkmark$ & $\checkmark$ & $\checkmark$ & $\checkmark$ \\
\hline Supplementary oxygen & $\checkmark$ & $\checkmark$ & $\checkmark$ & $\checkmark$ \\
\hline Mechanical ventilation & $\checkmark$ & $\checkmark$ & $\checkmark$ & $\checkmark$ \\
\hline Death & $\checkmark$ & $\checkmark$ & $\checkmark$ & $\checkmark$ \\
\hline COVID-19 treatment & $\checkmark$ & $\checkmark$ & $\checkmark$ & $\checkmark$ \\
\hline Immune biomarkers & $\checkmark$ & $\checkmark$ & $\checkmark$ & - \\
\hline Leukocyte phenotyping & $\checkmark$ & $\checkmark$ & $\checkmark$ & - \\
\hline Cortisol/prolactin levels & $\checkmark$ & $\checkmark$ & $\checkmark$ & $\checkmark$ \\
\hline Immunoglobulin profile & - & $\checkmark$ & - & - \\
\hline D-dimer & - & - & $\checkmark$ & - \\
\hline Thromboelastometry & - & - & $\checkmark$ & - \\
\hline Neutralizing antibodies & - & - & - & $\checkmark$ \\
\hline Metabolomic analysis & $\checkmark$ & $\checkmark$ & $\checkmark$ & $\checkmark$ \\
\hline Gene expression & - & $\checkmark$ & $\checkmark$ & - \\
\hline Neutrophils & - & - & $\checkmark$ & - \\
\hline
\end{tabular}

${ }^{\mathrm{a}}$ Indicates data was registered at this time point.

${ }^{\mathrm{b}}$ Indicates data was not registered at this time point.

\section{Timeline}

The TARGET protocol comprises two arms, referred to as the timeline (T)-branch and the days of hospitalization (D)-branch. For the purposes of this study, the T-branch refers to patients with mild/moderate forms that did not require hospitalization. The D-branch refers to patients with severe/critical forms who required hospital admission. Patients on the T-branch will be analyzed on days 0,9 , and 18 (T0, T9, and T18), while patients on the D-branch will be analyzed on days $0,3,9$, and 18 (D0, D3, D9, and D18; Figure 1).

\section{Biological Samples}

The TARGET protocol encompasses the collection of blood samples in four different types of containers. For these purposes, whole peripheral blood samples will be collected in vacuum tubes without an anticoagulant $(10 \mathrm{~mL})$ to obtain serum samples for metabolomic and biochemical analysis, in sodium citrate (5 $\mathrm{mL})$ for coagulation assays, and in heparin $(10 \mathrm{~mL})$ to obtain plasma samples and to isolate polymorphonuclear and peripheral blood mononuclear cells (PBMC). Samples will be collected at distinct time points as follows: day 0 , at inclusion by confirmation of COVID-19; day 3, within 2-4 days after inclusion; day 9, within 7-10 days after inclusion; and day 18, within 15-20 days after inclusion (Figure 2). 
Figure 2. Timeline showing the times of blood tests and the analysis performed. The upper line represents the duration of symptoms, a parameter used in the group of patients enrolled for prospective follow-up, composed primarily of patients who will evolve with mild conditions. The lower line represents the blood test dates in the hospitalized group: patients who have already entered the study with moderate to severe conditions, usually from the fifth day of symptom onset.

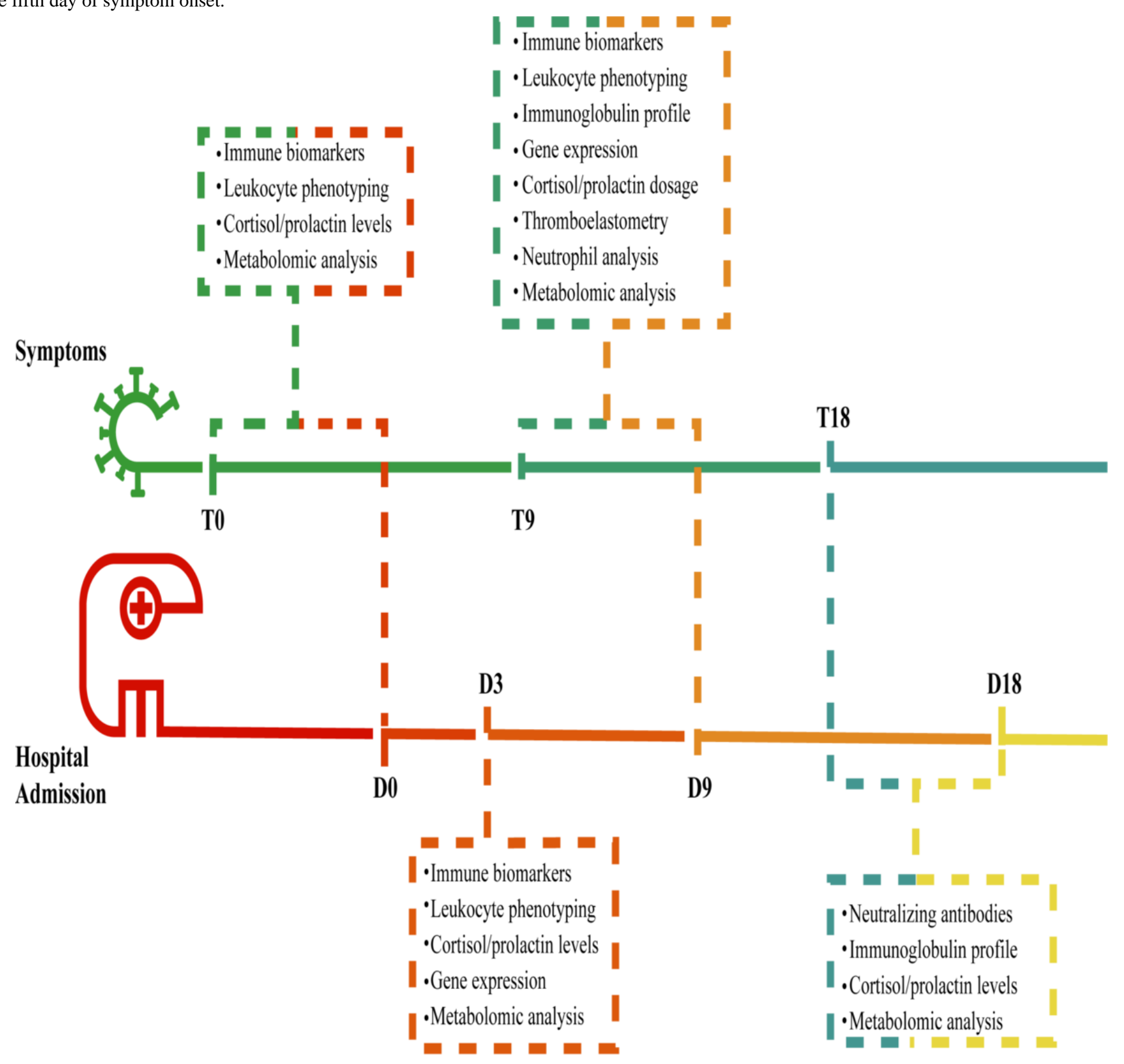

\section{Preanalytical Sample Handling and Preprocessing}

The whole blood samples will be processed to obtain serum or plasma samples and PBMC batches. Tubes will be centrifuged at $1900 \mathrm{~g}$ for 10 minutes at $22{ }^{\circ} \mathrm{C}$ to obtain serum or heparinized plasma and processed immediately within-facility for routine PBMC isolation and cryopreservation [17].

\section{Clinical Biochemistry and Hematologic Analysis}

Plasma samples obtained in ethylenediamine tetraacetic acid will be immediately analyzed at local clinical laboratory facilities. Biochemical analysis will be performed with a Rocas Cobas E411 system using compatible reagents to assess the levels of ferritin, troponin $\mathrm{T}$, creatine kinase, creatinine, aspartate aminotransferase, and alanine aminotransferase. All procedures will be performed according to the manufacturer's recommendations. Cortisol and prolactin in serum samples will be quantified at the Sabin Medicina Diagnóstica (Brasília, Brazil) using the chemiluminescent method (Siemens, Advia Centaur). Hematological analyses will be performed using whole blood obtained in sodium citrate tubes in an ABBOTT Cell Dyn 3700 analyzer or equivalent with samples from days 0,9 , and 18.

The coagulation profile will be assessed at the DASA Laboratory (Brasília, Brazil) by thromboelastometry tests carried out within a 3-hour interval after blood collection [13,25] as follows: extrinsic system assay (EXTEM), intrinsic system assay (INTEM), fibrinogen assay (FIBTEM), and nonactivated 
coagulation assay (NATEM; ROTEM, Werfen, Barcelona, Spain; Figure 3).

Figure 3. Thromboelastometry method for clot evaluation. A pin that spins around its own axis is put in contact with a citrated blood sample inside a cuvette. After recalcification and addition of a specific activator (depending upon the test), the clotting starts, and as it gets firmer, the spinning capacity of the axis is reduced, which is transformed by the system in a graphic representation of the clot, with increasing amplitude. As the fibrinolysis starts, the clot becomes less firm, which is represented as decreasing amplitude on the monitor. The extrinsic system assay (EXTEM)'s activator is thromboplastin and evaluates the extrinsic activation of coagulation. The intrinsic system assay's activator is elagic acid and evaluates the intrinsic activation of coagulation. The fibrinogen assay's activator is the same as EXTEM plus cytochalasin D, which inhibits platelet activity. This depicts only the participation of fibrinogen in the clot. The nonactivated coagulation assay is recalcified blood and is a nonactivated evaluation of coagulation. Circulating tissue factors, such as those expressed on monocytes in inflammatory states, will start the coagulation process. CT is the time frame from activation until an amplitude of $2 \mathrm{~mm}$ and represents thrombin formation. CFT represents the dynamic formation of thrombin and is the time frame between 2 mm and 20 $\mathrm{mm}$ of clot amplitude. A10 represents the clot amplitude 10 minutes after initiation and is directly related to MCF, enabling the clinic to make important decisions. MCF is the maximum amplitude of the clot and represents its main constituents, namely, fibrinogen and platelets. ML represents the percentage of clot reduction after initiation of fibrinolysis. Therefore, 60 minutes after initiation, thromboelastometry depicts important information about every phase of the coagulation process. A10: amplitude in 10 minutes; CFT: coagulation formation time; CT: coagulation time; MCF: maximum clot firmness; ML: maximum lysis.
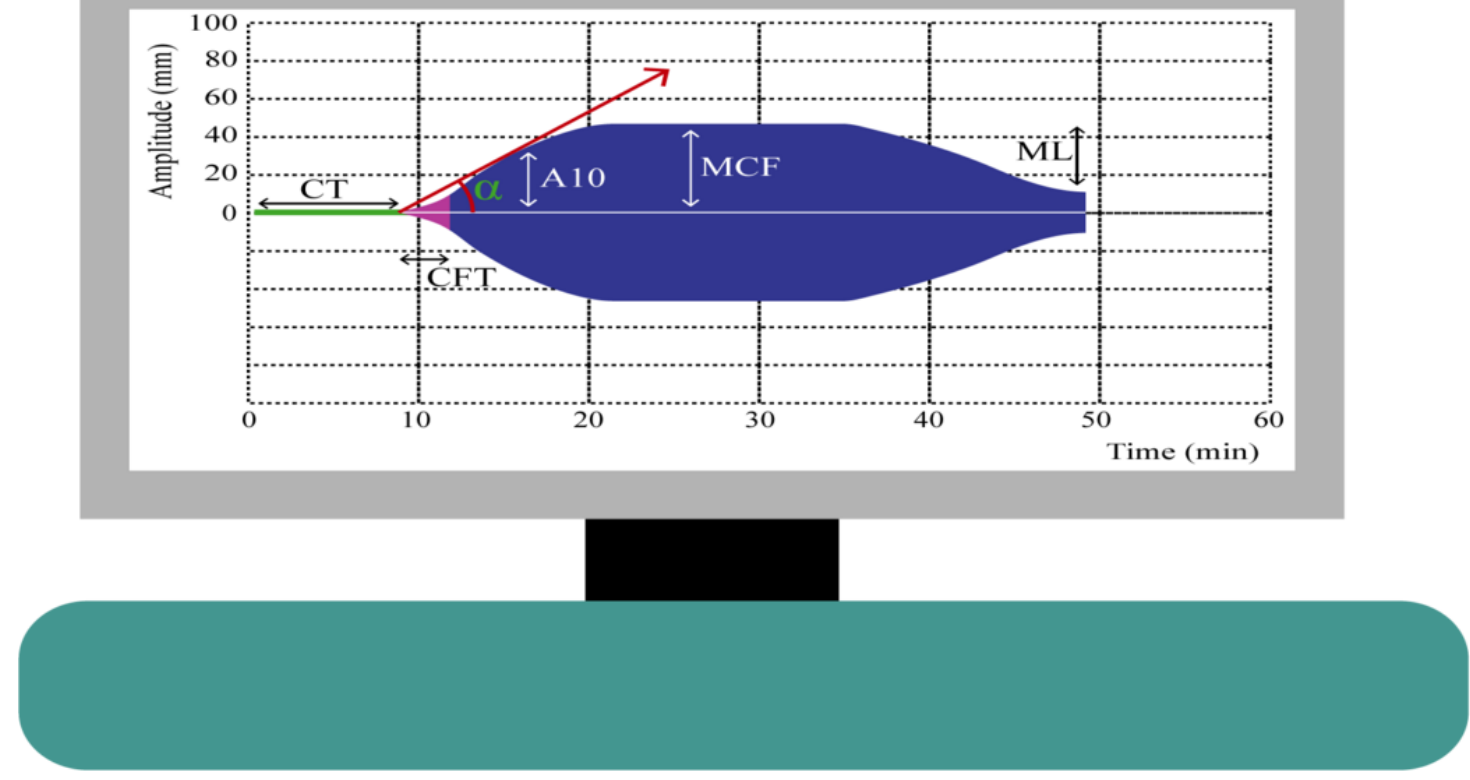

Oscillating axis $4.75^{\circ}$

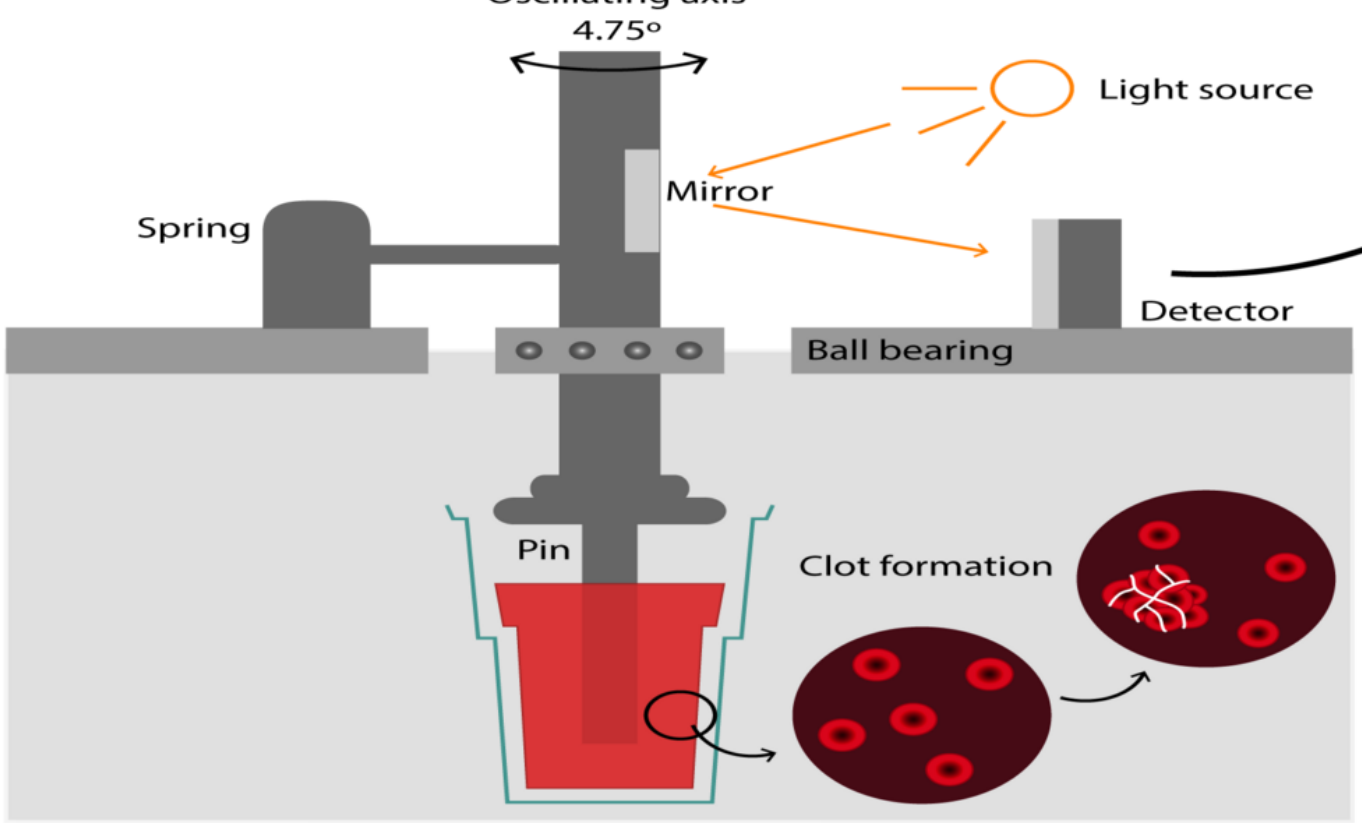




\section{Assessment of Molecular and Cellular Immunological Biomarkers}

Serum biomarkers will be assessed using the Luminex Bio-Plex Pro platform set for 27 human immune mediators (Bio-Rad Laboratories, California) for the simultaneous assessment of the following analytes: chemokines (CXCL8, CCL11, CCL3, CCL4, CCL2, CCL5, and CXCL10), proinflammatory cytokines (IL-1 $\beta$, IL-6, TNF- $\alpha$, IL-12p70, IFN- $\gamma$, IL-17A, and IL-15) and regulatory cytokines (IL-1Ra, IL-4, IL- 5, IL-9, IL-10, and IL-13), and cell growth factors (IL-2, IL-7, FGF-basic, PDGF, VEG, G-CSF, and GM-CSF). All procedures will follow the manufacturer's recommendations, with levels obtained at days 0,3 , and 9 .

The expression levels of immune mediators will be assessed by TaqMan quantitative RT-PCR (qRT-PCR) assays (Applied Biosystems, Foster City, CA) for each transcript, with retro-transcription set up with $1 \mathrm{ng} / \mu \mathrm{L}$ of RNA from PBMCs and from neutrophils. The reactions will be carried out on a QuantiStudio 3 Thermal Cycler (Applied Biosystems, CA) in standard mode [26]. Alternatively, qRT-PCR experiments for selected genes will be based on the SYBR Green (family of dyes for molecular biology) detection methodology using the SYBR Green PCR Master Mix and universal cycling conditions on the ABI Prism 7000 Sequence Detection System. Expression levels will be assessed on days 3 and 9 .

Immunophenotypic/functional profiles of circulating leukocytes will be assessed upon short-term culture in vitro. The cell suspension will be stained with distinct panels of monoclonal antibodies comprising CD3, CD4, CD8, HLA-DR, CD25, CD19, CD5, CD27, CD38, PD-1, CD28, CD14, CD16, and CD56 for cell surface analysis, and IL-1 $\beta$, IL-6, TNF- $\alpha$, IFN- $\alpha$, IL-5-PE, and IL-10 for evaluation of intracytoplasmic functional status. Cells will be run on a FORTESSA flow cytometer (BD Bioscience, CA). An average of 100,000 cells will be analyzed per sample. The phenotypic/functional parameters will be assessed using FlowJo software (BD, New Jersey). Leukocyte phenotyping will be obtained at days 0,3 , and 9 .

The immunoglobulin profile will be assessed by multiplexed PCR using cryopreserved PBMCs [19,27]. Sequencing libraries will be produced and sequenced using the Illumina MiSeq 2x300 bp platform. For each sample, 1 million reads corresponding to IgG and IgA will be produced. The reads will be processed using pRESTO [28], Ig heavy chain genes will be annotated using the MIXCR platform [29], and clonotypes will be determined as described in [30]. The immunoglobulin profile will be assessed in samples obtained on day 3 .

\section{Neutrophil Evaluation Experimental Design and Groups}

Neutrophils will be obtained from healthy donors and from patients at two different times, T9/T18 and D9/D18. Neutrophils isolated from each donor will be divided into two aliquots and incubated in buffer (control group) or phorbol 12-myristate 13-acetate (PMA; activated group). Cells from each group will be submitted to the functional and molecular analyses described in the following sections.

\section{Neutrophil Isolation and In Vitro Activation}

Cell isolation will be performed from peripheral venous blood by centrifugation in Percoll gradients, as previously described [27]. The remaining erythrocytes will be removed by hypotonic lysis. Samples containing $>95 \%$ neutrophils and $>98 \%$ viable neutrophils will be prepared in 50\% autologous plasma and divided into aliquots for control or activation in PMA [28].

\section{Evaluation of Reactive Oxygen Species Production and Phagocytosis}

The isolated neutrophils, after incubation in each condition, will be tested for reactive oxygen species production as described by Tahir et al [29].

\section{Evaluation of Neutrophil Extracellular Traps Production}

At the final 20 minutes of incubation in each condition, an aliquot of neutrophils will be removed and incubated with SYTOX green (high-affinity nucleic acid stain; $10 \mu \mathrm{mol} / \mathrm{L}$ ) and 4',6-diamidino-2-phenylindole (blue-fluorescent DNA stain; 1 $\mu \mathrm{g} / \mathrm{mL})$. Fluorescence readings will be performed both by fluorescence microscopy and on a spectrophotometer [30].

\section{Real-time Migration Assay}

Neutrophils from each condition will be applied to the upper chamber of a xCELLigence Real-Time Cell Analysis instrument and dynamically measured regarding the migration toward the chemoattractant N-Formylmethionyl-leucyl-phenylalanine (potent polymorphonuclear leukocyte chemotactic factor) [31].

\section{Neutrophil Proteomics}

Neutrophils obtained in all experimental conditions will be subjected to cell lysis by ultrasound cavitation and then trypsin digested. Eluted peptides will be subjected to capillary chromatography in a two-column online vented system. The fractions will be eluted directly into the ionization chamber of an Orbitrap Elite mass spectrometer to be analyzed in data-dependent acquisition mode. The 20 most intense ions will be selected for high-energy collision dissociation fragmentation, and the fragments will be analyzed in the ion-trap detector. Mass spectra will be analyzed for protein quantification and identification using the Progenesis and Peaks software packages, as well as in-house developed $\mathrm{R}$ scripts for statistical analysis [32].

\section{Metabolomic Profiling}

We will aim to analyze each serum sample collected from mild (T-branch group) and severe (D-branch group) case patients and healthy volunteers at each time point in the same manner (Figures 1 and 2). Untargeted metabolomics will be performed using a Bruker UPLC-PDA-QqTOF (ultra-performance liquid chromatography photodiode array quadrupole-quadrupole-time-of-flight) compact (UPLC-MS/MS). The obtained data will be used to build molecular networking using the Global Natural Products Social Molecular Networking platform, which gives a better overview of the metabolic profile and group compound classes in clusters. For statistical validation of the metabolite differences between the groups, the data will be processed in MZmine and analyzed 
by means of chemometric tools, as provided by the MetaboAnalyst platform. Based on this broad chemical outline, specific metabolites will be quantitatively analyzed using targeted metabolomics using a CLAE Quadrupole/Quadrupole. This approach will be used to validate and quantify specific components of interest, such as metabolites involved in the glycolytic and hexosamine pathways, and tricarboxylic acid cycle, among other possible biomarkers.

\section{Sample Size Calculation}

The sample size calculation was based on the assumption that $20 \%$ of patients who are critically ill have elevated TNF- $\alpha$ levels compared to $5 \%$ of patients who are noncritically ill [8]. TNF levels will be normalized according to the study population. Based on a bilateral $95 \%$ significance level, a power of $80 \%$, and a case-control ratio of 1, a sample size of 200 patients can be predicted.

\section{Statistical Analysis}

Clinical and demographic data between groups of patients (severe vs nonsevere) as well as treatment profiles and differences in successive time points will be compared. To compare mean values of soluble biomarkers between groups, the $t$ student test (or analysis of variance with post hoc test for multiple groups) or the nonparametric Mann-Whitney (or Kruskal-Wallis for multiple groups) test will be used as appropriate, according to the observed sample distribution features. The SAS 9.3 software (SAS Institute Inc) will be used with a significance level set at .05 . The main binary outcomes will be analyzed by the estimation of relative risks using a log-binomial generalized model or by the area under the curve using trapezoidal methods. All personnel conducting laboratory procedures will be blinded regarding the patient status (severity of COVID-19). Receiver operating characteristic curves will determine cutoff points at which individual or combined scores of the molecular and cellular biomarkers become informative of the progression of COVID-19 cases, according to optimal sensitivity and specificity to identify the signature that best characterize each clinical status.

\section{Results}

This protocol is in the data collection phase. Study recruitment started in June 2020. At the end of July 2020, 30 T-branch and 85 D-branch patients were enrolled.

We expected to complete all patient inclusion by June 2020, but with the continuous increase in COVID-19 cases in Brazil, the authors decided to continue including patients until February 2021.

Data analysis is scheduled to start after all inclusion data have been collected.

The coagulation study branch, with thromboelastometry, has already been completed. Preliminary results confirm deregulation in initiation of the hemostasis process. The results are currently being analyzed and prepared for publication.

\section{Discussion}

This TARGET protocol was designed to standardize the evaluation of cellular and molecular immune biomarkers to evaluate their potential as prognosis tools during COVID-19 infection at different degrees of clinical severity. As the host immune response impacts COVID-19 progression, understanding the behavior of immune biomarkers at the different phases of the illness constitutes a crucial step in the identification of patients who will deteriorate and those who will benefit most from treatment. A simultaneous comprehensive assessment of the different aspects of the immune response was the approach adopted for this protocol.

An early study in China showed that the elevation of certain biomarkers such as D-dimer, together with inflammatory markers including ferritin, was associated with the worst outcomes after SARS-CoV-2 infection [33]. These measurements have been incorporated in some clinical practice centers, but their course during disease and actual value in prognosis are not yet fully described. Higher levels of a series of other interleukins were also described in severe forms of the disease [34], contributing to the concept of a cytokine storm, but their exact significance remains unclear. A thorough evaluation of different immune aspects could help to clarify their role.

Less information regarding the cellular immune response has been published, but its role is progressively gaining more attention from the scientific community, as some evidence notes that humoral immunity does not appear to be sufficiently developed after the first SARS-CoV-2 infection [35]. A simultaneous evaluation of these two kinds of immune responses in the TARGET study could help to understand this interface.

Thromboembolism is a recurrent finding among patients with COVID-19, which has made thromboprophylaxis a vital part of the available supportive treatment options. Unfortunately, there is considerable uncertainty about the degree of coagulation derangement, the possible association with the inflammatory response elicited including neutrophil extracellular traps [36], and the consequences of these alterations [13]. Further knowledge is needed to better understand COVID-19-associated coagulopathy to provide frontline clinicians with better treatment options to manage the ensuing clinical manifestations.

A metabolomics study of serum from patients with COVID-19 annotated 941 metabolites. The authors identified metabolic patterns in severe cases that could be used to propose diagnostic models capable of predicting progression to severe infection and assist in the treatment strategy [37]. Observed changes in the lipid profile (>100 lipids) cause liver damage, as reflected by abnormal serum levels of bilirubin and bile acids [37]. Another study revealed the consequences of treating patients with SARS-CoV with methylprednisolone. Disturbances in the serum lipid profile 12 years post infection were associated with long-term systemic damage caused by high doses of methylprednisolone [38]. Metabolomics is therefore a powerful tool for identifying and monitoring the prognosis and markers at different stages of a disease, particularly for unknown infections [24]. 
Similarly, neutrophil functional and molecular assays have been performed in patients with COVID-19 [39], clearly indicating the participation of these cells in the pathogeny, although no specific molecular model has yet been proposed [40]. Therefore, the proteomic approach coupled with further analysis at the transcription and functional level will provide evidence to correlate neutrophil activity to the disease severity and time course.

Only two treatments have shown substantial beneficial effects to date in randomized trials in COVID-19, with both exhibiting heterogeneity of treatment effect between subgroups. A preliminary report from Beigel et al [41] showed that remdesivir shortened the median recovery in a trial from 15 days in the placebo group to 11 days, but those on mechanical ventilation were less likely to benefit. On the other hand, dexamethasone demonstrated a reduction in mortality at 28 days, but mainly in those on mechanical ventilation [42]. One possible explanation for these findings is that an adequate immune response to stop viral replication is needed at the onset of the disease. The excess of inflammation presenting is detrimental. As the time when these treatments are initiated could be key, immune biomarkers that signal phases of disease to guide the timing of treatments could be extremely useful in clinical practice.

In summary, TARGET is a translational study that aims to verify the role of cellular and molecular immune biomarkers in the prognosis of COVID-19, opening up avenues for their use in clinical practice. The results that will be achieved are not meant to be restricted to the description of the disease pathophysiology, but to form a basis for future clinical studies that will ultimately help the clinicians on the front line.

\section{Acknowledgments}

The authors thank the financial support provided by Conselho Nacional de Desenvolvimento Científico e Tecnológico (CNPq), Coordenação de Aperfeiçoamento de Pessoal de Nível Superior (CAPES), under process no. 88887.506611/2020-00 and 88887.504420/2020-00 and Fundação de Amparo à Pesquisa de Minas Gerais (FAPEMIG), under process no. REDE-00140-16. The authors thank the ArboControl Brazil Project funded by the Ministry of Health, under process no. TED 74/2016 and TED 42/2017 for financial support. The study has been supported by Hospital Universitário de Brasília (HuB) from Universidade de Brasília (UnB), Hospital Regional da Asa Norte (HRAN). LAN, LFF, OTN, WF and OAMF thank the CNPq for PQ-fellowship Program. The funders had no contribution in the study design, data collection and analysis, decision to publish or manuscript preparation. We thank the "Nucleus of Support on Research from Institute Sabin - Brasília for performing hormonal tests and sample pre-analytical handling, the DASA Laboratory for performing the ROTEM analysis and the Werfen Medical for supplying reagents for ROTEM analysis.

\section{Conflicts of Interest}

None declared.

\section{References}

1. Guo Y, Cao Q, Hong Z, Tan Y, Chen S, Jin H, et al. The origin, transmission and clinical therapies on coronavirus disease 2019 (COVID-19) outbreak - an update on the status. Mil Med Res 2020 Mar 13;7(1):11 [FREE Full text] [doi: 10.1186/s40779-020-00240-0] [Medline: 32169119]

2. Wang TT, Moon HS, Le A, Panchal N. Proceedings of the OMS COVID-19 response conference. J Oral Maxillofac Surg 2020 Aug;78(8):1268-1274 [FREE Full text] [doi: 10.1016/j.joms.2020.05.003] [Medline: 32422192]

3. Yang Y, Peng F, Wang R, Yange M, Guan K, Jiang T, et al. The deadly coronaviruses: the 2003 SARS pandemic and the 2020 novel coronavirus epidemic in China. J Autoimmun 2020 May;109:102434 [FREE Full text] [doi: 10.1016/j.jaut.2020.102434] [Medline: 32143990]

4. Aggarwal S, Garcia-Telles N, Aggarwal G, Lavie C, Lippi G, Henry BM. Clinical features, laboratory characteristics, and outcomes of patients hospitalized with coronavirus disease 2019 (COVID-19): early report from the United States. Diagnosis (Berl) 2020 May 26;7(2):91-96. [doi: 10.1515/dx-2020-0046] [Medline: 32352401]

5. Conner SD, Schmid SL. Identification of an adaptor-associated kinase, AAK1, as a regulator of clathrin-mediated endocytosis. J Cell Biol 2002 Mar 04;156(5):921-929 [FREE Full text] [doi: 10.1083/jcb.200108123] [Medline: 11877461]

6. Verdecchia P, Cavallini C, Spanevello A, Angeli F. The pivotal link between ACE2 deficiency and SARS-CoV-2 infection. Eur J Intern Med 2020 Jun;76:14-20 [FREE Full text] [doi: 10.1016/j.ejim.2020.04.037] [Medline: 32336612]

7. Sodhi CP, Wohlford-Lenane C, Yamaguchi Y, Prindle T, Fulton WB, Wang S, et al. Attenuation of pulmonary ACE2 activity impairs inactivation of des-Arg bradykinin/BKB1R axis and facilitates LPS-induced neutrophil infiltration. Am J Physiol Lung Cell Mol Physiol 2018 Jan 01;314(1):L17-L31 [FREE Full text] [doi: 10.1152/ajplung.00498.2016] [Medline: 28935640]

8. Huang C, Wang Y, Li X, Ren L, Zhao J, Hu Y, et al. Clinical features of patients infected with 2019 novel coronavirus in Wuhan, China. Lancet 2020 Feb 15;395(10223):497-506 [FREE Full text] [doi: 10.1016/S0140-6736(20)30183-5] [Medline: 31986264]

9. Mehta P, McAuley DF, Brown M, Sanchez E, Tattersall RS, Manson JJ, HLH Across Speciality Collaboration, UK. COVID-19: consider cytokine storm syndromes and immunosuppression. Lancet 2020 Mar 28;395(10229):1033-1034 [FREE Full text] [doi: 10.1016/S0140-6736(20)30628-0] [Medline: 32192578] 
10. Wu D, Yang XO. TH17 responses in cytokine storm of COVID-19: an emerging target of JAK2 inhibitor Fedratinib. J Microbiol Immunol Infect 2020 Jun;53(3):368-370 [FREE Full text] [doi: 10.1016/j.jmii.2020.03.005] [Medline: 32205092]

11. Bennardo F, Buffone C, Giudice A. New therapeutic opportunities for COVID-19 patients with Tocilizumab: possible correlation of interleukin-6 receptor inhibitors with osteonecrosis of the jaws. Oral Oncol 2020 Jul;106:104659 [FREE Full text] [doi: 10.1016/j.oraloncology.2020.104659] [Medline: 32209313]

12. RECOVERY Collaborative Group, Horby P, Lim WS, Emberson JR, Mafham M, Bell JL, et al. Dexamethasone in hospitalized patients with Covid-19. N Engl J Med 2021 Feb 25;384(8):693-704 [FREE Full text] [doi: 10.1056/NEJMoa2021436] [Medline: $\underline{\text { 32678530] }}$

13. Mortus JR, Manek SE, Brubaker LS, Loor M, Cruz MA, Trautner BW, et al. Thromboelastographic results and hypercoagulability syndrome in patients with coronavirus disease 2019 who are critically ill. JAMA Netw Open 2020 Jun 01;3(6):e2011192 [FREE Full text] [doi: 10.1001/jamanetworkopen.2020.11192] [Medline: 32501489]

14. Zhang C, Wu Z, Li J, Zhao H, Wang G. Cytokine release syndrome in severe COVID-19: interleukin-6 receptor antagonist tocilizumab may be the key to reduce mortality. Int J Antimicrob Agents 2020 May;55(5):105954 [FREE Full text] [doi: 10.1016/j.ijantimicag.2020.105954] [Medline: 32234467]

15. Xu X, Han M, Li T, Sun W, Wang D, Fu B, et al. Effective treatment of severe COVID-19 patients with tocilizumab. Proc Natl Acad Sci U S A 2020 May 19;117(20):10970-10975 [FREE Full text] [doi: 10.1073/pnas.2005615117] [Medline: 32350134]

16. von Elm E, Altman DG, Egger M, Pocock SJ, Gøtzsche PC, Vandenbroucke JP, STROBE Initiative. The Strengthening the Reporting of Observational Studies in Epidemiology (STROBE) Statement: guidelines for reporting observational studies. Int J Surg 2014 Dec;12(12):1495-1499 [FREE Full text] [doi: 10.1016/j.ijsu.2014.07.013] [Medline: 25046131]

17. Fuss IJ, Kanof ME, Smith PD, Zola H. Isolation of whole mononuclear cells from peripheral blood and cord blood. Curr Protoc Immunol 2009 Apr;Chapter 7:Unit7.1. [doi: 10.1002/0471142735.im0701s85] [Medline: 19347849]

18. Favalli EG, Biggioggero M, Maioli G, Caporali R. Baricitinib for COVID-19: a suitable treatment? Lancet Infect Dis 2020 Sep;20(9):1012-1013 [FREE Full text] [doi: 10.1016/S1473-3099(20)30262-0] [Medline: 32251638]

19. McDaniel JR, DeKosky BJ, Tanno H, Ellington AD, Georgiou G. Ultra-high-throughput sequencing of the immune receptor repertoire from millions of lymphocytes. Nat Protoc 2016 Mar;11(3):429-442. [doi: 10.1038/nprot.2016.024] [Medline: 26844430]

20. Yang PL. Chapter 14 - metabolomics and lipidomics: yet more ways your health is influenced by fat. In: Katze MG, Korth MJ, Law GL, Nathanson N, editors. Viral Pathogenesis: From Basics to Systems Biology. Cambridge, MA: Academic Press; 2016:181-198.

21. Santos MCD, Morais CLM, Nascimento YM, Araujo JMG, Lima KMG. Spectroscopy with computational analysis in virological studies: a decade (2006-2016). Trends Analyt Chem 2017 Dec;97:244-256 [FREE Full text] [doi: 10.1016/j.trac.2017.09.015] [Medline: 32287542]

22. Deng W, Rao J, Chen X, Li D, Zhang Z, Liu D, et al. Metabolomics study of serum and urine samples reveals metabolic pathways and biomarkers associated with pelvic organ prolapse. J Chromatogr B Analyt Technol Biomed Life Sci 2020 Jan 01;1136:121882. [doi: 10.1016/j.jchromb.2019.121882] [Medline: 31809960]

23. Kouskoumvekaki I, Panagiotou G. Navigating the human metabolome for biomarker identification and design of pharmaceutical molecules. J Biomed Biotechnol 2011;2011. [doi: 10.1155/2011/525497] [Medline: 20936122]

24. Martín-Blázquez A, Díaz C, González-Flores E, Franco-Rivas D, Jiménez-Luna C, Melguizo C, et al. Untargeted LC-HRMS-based metabolomics to identify novel biomarkers of metastatic colorectal cancer. Sci Rep 2019 Dec 27;9(1):20198. [doi: 10.1038/s41598-019-55952-8] [Medline: $\underline{\text { 31882610] }}$

25. Calatzis AGK, Spannagl M, Vorweg M. Targeted treatment of acute haemostatic disorders. ROTEM Analysis. URL: https:/ /www.rotem.de/wp-content/uploads/2016/09/ROTEM_Analysis_delta_platelet_EN_2016-09-website.pdf [accessed 2020-07-20]

26. Zhang T, Li L, Shang Q, Lv C, Wang C, Su B. Circulating miR-126 is a potential biomarker to predict the onset of type 2 diabetes mellitus in susceptible individuals. Biochem Biophys Res Commun 2015;463(1-2):60-63. [doi: 10.1016/j.bbrc.2015.05.017] [Medline: 25986735]

27. Carvalho LO, Aquino EN, Neves ACD, Fontes W. The neutrophil nucleus and its role in neutrophilic function. J Cell Biochem 2015 Sep;116(9):1831-1836. [doi: 10.1002/jcb.25124] [Medline: 25727365]

28. Aquino EN, Neves ACD, Santos KC, Uribe CE, Souza PEN, Correa JR, et al. Proteomic analysis of neutrophil priming by PAF. Protein Pept Lett 2016;23(2):142-151. [doi: 10.2174/0929866523666151202210604] [Medline: 26631175]

29. Tahir M, Arshid S, Fontes B, Castro MS, Luz IS, Botelho KLR, et al. Analysis of the effect of intestinal ischemia and reperfusion on the rat neutrophils proteome. Front Mol Biosci 2018;5:89. [doi: 10.3389/fmolb.2018.00089] [Medline: 30555831]

30. Hule GP, Bargir UA, Kulkarni M, Kambli P, Taur P, Desai M, et al. Does pioglitazone lead to neutrophil extracellular traps formation in chronic granulomatous disease patients? Front Immunol 2019;10:1739. [doi: 10.3389/fimmu.2019.01739] [Medline: $\underline{31428088]}$

31. Cano PM, Vargas A, Lavoie J. A real-time assay for neutrophil chemotaxis. Biotechniques 2016;60(5):245-251 [FREE Full text] [doi: 10.2144/000114416] [Medline: 27177817] 
32. Cury N, Silva R, Andre M, Fontes W, Ricart C, Castro M, et al. Root proteome and metabolome reveal a high nutritional dependency of aluminium in Qualea grandiflora Mart. (Vochysiaceae). Plant Soil 2019 Nov 14;446(1-2):125-143. [doi: 10.1007/s11104-019-04323-3]

33. Zhou F, Yu T, Du R, Fan G, Liu Y, Liu Z, et al. Clinical course and risk factors for mortality of adult inpatients with COVID-19 in Wuhan, China: a retrospective cohort study. Lancet 2020 Mar 28;395(10229):1054-1062 [FREE Full text] [doi: 10.1016/S0140-6736(20)30566-3] [Medline: 32171076]

34. Velavan TP, Meyer CG. Mild versus severe COVID-19: laboratory markers. Int J Infect Dis 2020 Jun;95:304-307 [FREE Full text] [doi: 10.1016/j.ijid.2020.04.061] [Medline: 32344011]

35. Siracusano G, Pastori C, Lopalco L. Humoral immune responses in COVID-19 patients: a window on the state of the art. Front Immunol 2020;11:1049. [doi: 10.3389/fimmu.2020.01049] [Medline: 32574261]

36. Middleton EA, He X, Denorme F, Campbell RA, Ng D, Salvatore SP, et al. Neutrophil extracellular traps contribute to immunothrombosis in COVID-19 acute respiratory distress syndrome. Blood 2020 Sep 03;136(10):1169-1179 [FREE Full text] [doi: 10.1182/blood.2020007008] [Medline: 32597954]

37. Song L, Yin Q, Kang M, Ma N, Li X, Yang Z, et al. Untargeted metabolomics reveals novel serum biomarker of renal damage in rheumatoid arthritis. J Pharm Biomed Anal 2020 Feb 20;180:113068. [doi: 10.1016/j.jpba.2019.113068] [Medline: 31884392]

38. Wu Q, Zhou L, Sun X, Yan Z, Hu C, Wu J, et al. Altered lipid metabolism in recovered SARS patients twelve years after infection. Sci Rep 2017 Aug 22;7(1):9110. [doi: 10.1038/s41598-017-09536-z] [Medline: 28831119]

39. Didangelos A. COVID-19 hyperinflammation: what about neutrophils? mSphere 2020 Jun 24;5(3). [doi: 10.1128/mSphere.00367-20] [Medline: 32581077]

40. Laforge M, Elbim C, Frère C, Hémadi M, Massaad C, Nuss P, et al. Tissue damage from neutrophil-induced oxidative stress in COVID-19. Nat Rev Immunol 2020 Sep;20(9):515-516 [FREE Full text] [doi: 10.1038/s41577-020-0407-1] [Medline: 32728221]

41. Beigel JH, Tomashek KM, Dodd LE, Mehta AK, Zingman BS, Kalil AC, ACTT-1 Study Group Members. Remdesivir for the treatment of Covid-19 - final report. N Engl J Med 2020 Nov 05;383(19):1813-1826 [FREE Full text] [doi: 10.1056/NEJMoa2007764] [Medline: 32445440]

42. RECOVERY Collaborative Group. Dexamethasone for COVID-19: preliminary findings. Drug Ther Bull 2020 Sep;58(9):133. [doi: 10.1136/dtb.2020.000045] [Medline: 32690491]

\section{Abbreviations}

ACE2: angiotensin converting enzyme-2

CNPq: Conselho Nacional de Desenvolvimento Científico e Tecnológico

D: days of hospitalization

EXTEM: extrinsic system assay

FIBTEM: fibrinogen assay

IFN- $\gamma$ : interferon-gamma

INTEM: intrinsic system assay

MCP-1: monocyte chemoattractant protein-1

NATEM: nonactivated coagulation assay

PBMC: peripheral blood mononuclear cells

PMA: phorbol 12-myristate 13-acetate

qRT-PCR: quantitative reverse transcription polymerase chain reaction

RECOVERY: Randomised Evaluation of COVID-19 Therapy

RT-PCR: reverse transcription polymerase chain reaction

SARS-CoV: severe acute respiratory syndrome-related coronavirus

STROBE: Strengthening the Reporting of Observational Studies in Epidemiology

T: timeline

TNF- $\alpha$ : tumor necrosis factor-alpha

UnB: University of Brasília

UPLC-PDA-QqTOF: ultra-performance liquid chromatography photodiode array quadrupole-quadrupole-time-of-flight

WHO: World Health Organization 
Edited by G Eysenbach; submitted 09.09.20; peer-reviewed by A Allam, E Soto Vega; comments to author 02.12.20; revised version received 13.12.20; accepted 19.02.21; published 04.03.21

Please cite as:

Kurizky P, Nóbrega OT, Soares AADSM, Aires RB, Albuquerque CPD, Nicola AM, Albuquerque P, Teixeira-Carvalho A, Naves LA, Fontes W, Luz IS, Felicori L, Gomides APM, Mendonça-Silva DL, Espindola LS, Martins-Filho OA, de Lima SMB, Mota LMH, Gomes $C M$

Molecular and Cellular Biomarkers of COVID-19 Prognosis: Protocol for the Prospective Cohort TARGET Study

JMIR Res Protoc 2021;10(3):e24211

URL: https://www.researchprotocols.org/2021/3/e24211

doi: $10.2196 / 24211$

PMID: 33661132

CPatricia Kurizky, Otávio T Nóbrega, Alexandre Anderson De Sousa Munhoz Soares, Rodrigo Barbosa Aires, Cleandro Pires De Albuquerque, André Moraes Nicola, Patrícia Albuquerque, Andréa Teixeira-Carvalho, Luciana Ansaneli Naves, Wagner Fontes, Isabelle Souza Luz, Liza Felicori, Ana Paulo Monteiro Gomides, Dayde Lane Mendonça-Silva, Laila Salmen Espindola, Olindo Assis Martins-Filho, Sheila Maria Barbosa de Lima, Licia Maria Henrique Mota, Ciro Martins Gomes. Originally published in JMIR Research Protocols (http://www.researchprotocols.org), 04.03.2021. This is an open-access article distributed under the terms of the Creative Commons Attribution License (https://creativecommons.org/licenses/by/4.0/), which permits unrestricted use, distribution, and reproduction in any medium, provided the original work, first published in JMIR Research Protocols, is properly cited. The complete bibliographic information, a link to the original publication on http://www.researchprotocols.org, as well as this copyright and license information must be included. 